\title{
REAL-TIME AND PARALLEL QUALITY CONTROL PROCESSING ON INDUSTRIAL PRODUCTION LINES
}

\author{
Norbert VARJASI \\ Department of Information Technology, Széchenyi István University Győr \\ H-9026 Győr, Egyetem tér 1, Hungary, e-mail: varjasin@sze.hu
}

Received 12 February 2008; accepted 30 April 2008

\begin{abstract}
The subject of the article is the evaluation of three acoustical algorithms on industrial production lines. The algorithm is used for the inspection of work-pieces in a noisy industrial environment. High efficiency performance requirements restricted running time and expected precise results make these inspections problematic. A sequential and two parallel algorithms and processing methods for these measurements were developed. These algorithms are real-time and multi-channel processing methods on industrial production lines. They carry out series of precision measurements in the time frame available. After the inspection the quality class and usability of the pieces are determined. These computer-aided inspections are more effective than human measurements because they are more precise, can be repeated multiple times, even with different quality requirements. They are usable on high-speed industrial production lines to obtain results in digital format.
\end{abstract}

Keywords: Industrial production line, Parallel processing, Noise wavelet

\section{Computer-aided and human inspections}

Manufacturers used human inspections before but they had several problems with it. It required well-qualified workers who have sharp ears, and special silent rooms near the production line. These inspections are expensive (cost and time) and only several randomly selected work pieces can be inspected. The quality control categories have subjective values. The human senses are troublesome, and inspection workers may change at every hour.

With computer-aided inspections the precision of the measurements is better, and the silent room is not needed. The measurement sequence can be repeated multiple 
times, even with different quality requirements. These inspections are available at the high-speed industrial lines and every work-piece can be inspected [1]. With computeraided inspections we look forward to decreasing the number of rejected work-pieces and consumer's refusals.

\section{The inspection workspace}

The inspection of work-pieces happens in a high noise industrial environment. The measurement hardware is a specialized part of the industrial assembly lines (Fig. 1). At this point a complex noise test of work-pieces is performed for quality inspection. The inspection is synchronized with the other phases of the production line [2], with line balancing [3].

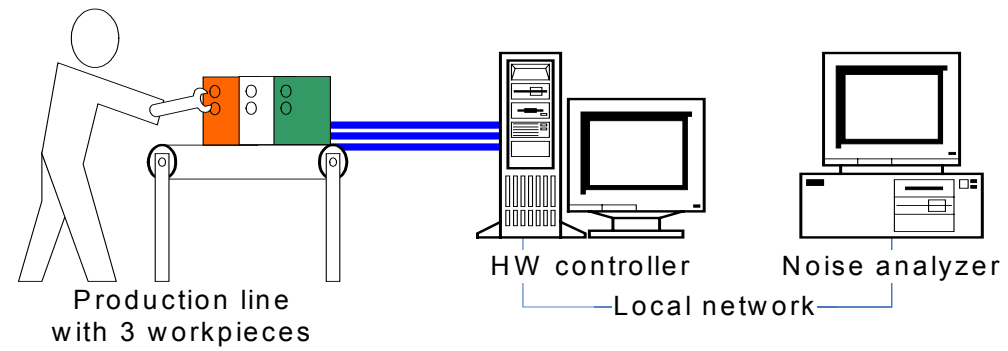

Fig. 1. A noise inspection workspace at the production line

The operator's role is to identify the work-piece with its bar code and to insert the piece into the hardware. The automatic measure station checks the mechanical noise of piece in restricted running time (see Fig. 2 and Fig. 3). At the end of procedure the operator reads the result from the computer screen and fits it into one of the predefined quality classes. Finally he inserts a new work-piece.

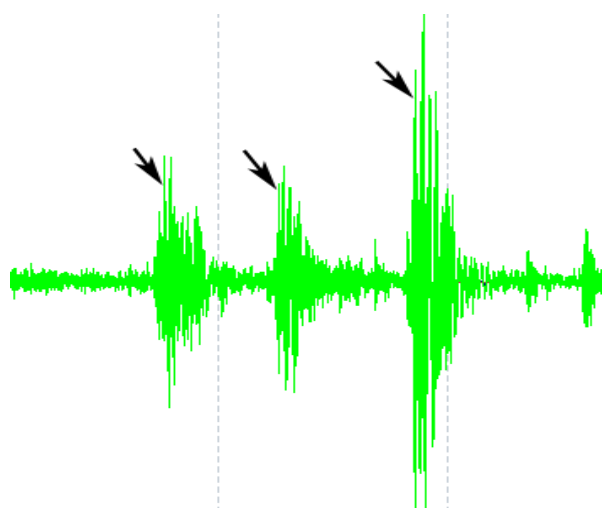

Fig. 2. A work-piece with periodic noise, bad quality 


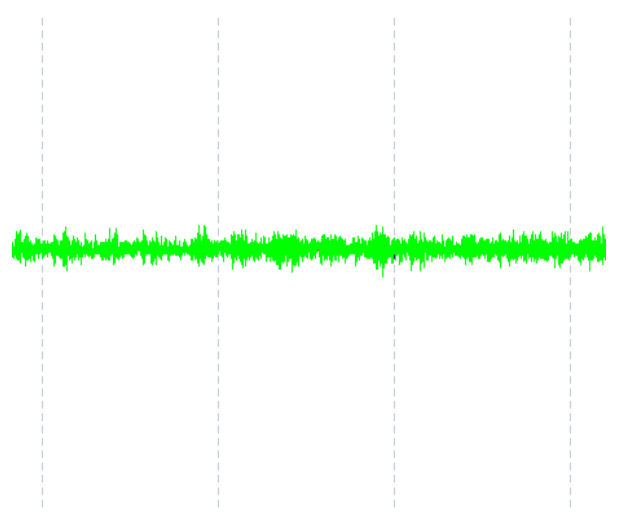

Fig. 3. A good quality work-piece's wavelet

The measurement tool has three parts: the specialized hardware, the control program and the noise analyzer software. This software is run on a multiprocessor computer or two independent PCs.

The role of the control program is to actuate the hardware with low-level functions (such as electric power and air pressure control, sensors state detecting, the amplifier and noise detector moving etc.) and to notify the operator.

\subsection{The efficiency of measurement}

On the industrial production line the inspection and evaluation time is strictly restricted by the production line's cycle time [2]. Every short period of wasted time neglected that was at design time becomes much more significant at a daily or weekly operation. To take the estimated annual cost of operation into consideration we have to minimize the running time. In our case real time noise analysis is a complex measurement. The efficiency of the method is better when the idle time of the inspection routine can be decreased.

With the analyzer software the quality class is determined in a few steps. First the wavelet's precision, noise level and the start-stop signals are detected. If the sample is good a deep and comprehensive analysis is started, otherwise the inspection is repeated.

\subsection{Parallel methods}

At the first model a sequential inspection routine of the work-pieces was tried. According to precedent similar inspections [4], these noise inspection problems are traditionally linear and sequential problems.

In our case the running time of these algorithms is long and it can only be used in a laboratory environment. These inspections are eight times slower than the production line's cycle time. The hardware is waiting for a long time while the inspection routine is running. In some cases, when the noisy wavelets cannot be evaluated, the waiting time is longer then cycle time. At a continuous assembly line this is not permissible. 
With a parallel method the program divides this process into several asynchronous phases/stages. The hardware inspection is continuous while the analyzer software evaluates the noise tests. The manufacturer's claims are minimal time waste and the possibility of three independent work-piece inspections at the same time (see Fig. 1). The maximal inspection time should be less then or equal to cycle time (approximately $20 \mathrm{~s})$.

\subsection{The noise analysis}

The noise analyzer software saves the characteristic noise of the moving parts of the work-piece at every stage of the inspection. The sampling rate of these inspections is high $(200 \mathrm{kHz})$ to detect every little inaudible malfunction. Thus these wavelets are large.

The wavelet inspection consists of two steps. First a very fast pre-tester heuristic algorithm detects if the work-piece has one of the several main errors. After this fast inspection a deep and comprehensive measurement starts to specify the usability of piece and the quality class (see Fig. 4).

The pre-test gives 'immediate' result between 10 and 100 milliseconds, and the waiting time of the measurement hardware is easily minimized. If the measurement is noisy or the pre-test was false the measurement must be repeated. Through the pre-test the wavelets were inspected with background noise analysis [5], peak detection and sound pressure [1].

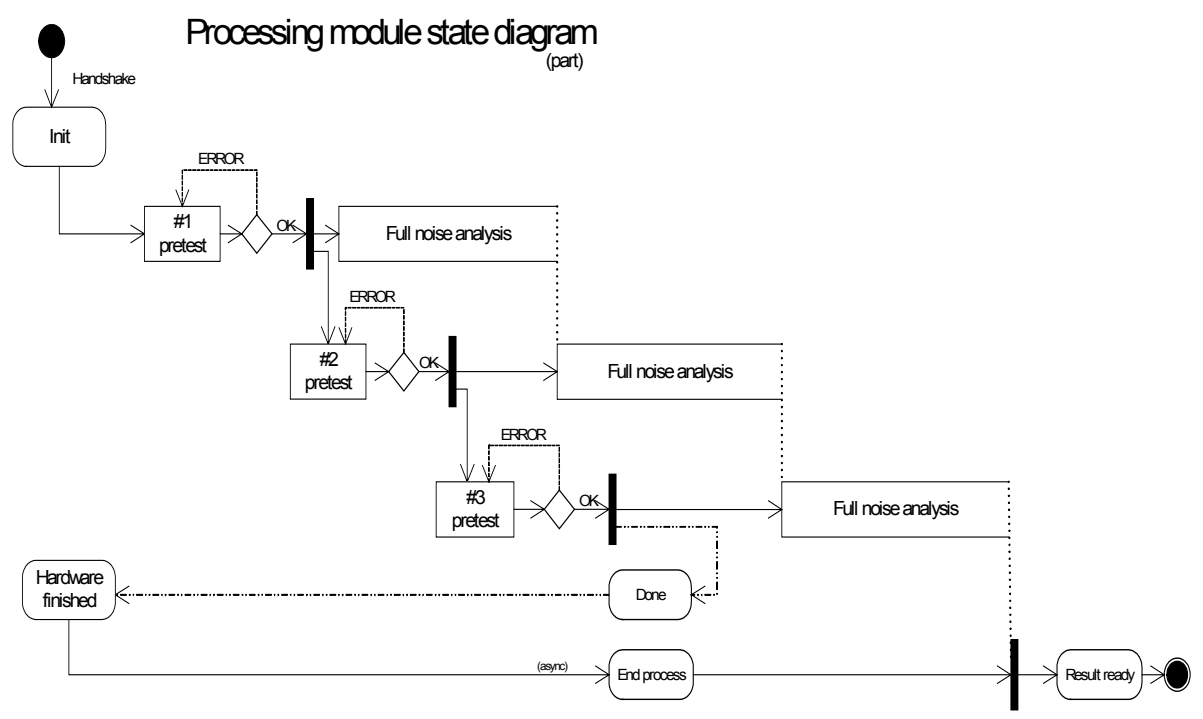

Fig. 4. State diagram of the analyzer software with three repeats

After the successful pre-test we inspect the full wavelet. First some decomposition is needed. With spectral analysis the signals from the noises are divided [6] and fast Fourier transformation is used [1]. After that a heuristic inspection is taken based on the 
Zwicker model [7]. With this method the whole frequency area is processed and the low level signals are eliminated [5], [8]. From this result the periodic and clatter noise can be found easily. Finally the quality of the work-piece can be determined. (In the future if every part's dimension and parameter is known the part with a malfunction could be detected.)

\subsection{Improved parallel algorithm}

These inspections run in a noisy industrial environment and a repeatable algorithm was developed. This algorithm is able to repeat some smaller phases of the inspection routine. According to the manufacturer the tool was improved with some communicational and controller functions. After these developments idle time was shortened, and shorter processing time was achieved for a work-piece.

\section{Results}

To illustrate the efficiency of parallel algorithms on industrial production lines the cycle time is used. From this respect the measurement has three phases. The first one is the initialization time while the operator changes the work-pieces at the station. This is a manufacturer's standard time and it can be neglected. (See the diagram at initialize time Fig. 5 and Fig. 6.)

The second part is the idle time, while the hardware waits for instructions. The third part is the working time, while the hardware inspection is running. Every test was run with a hundred work-pieces. The running times above are average values.

The first method was the sequential method. The maximal repeat of inspections was three and the full processing time was $136 \mathrm{~s}$ (see Fig. 5) because of the noisy environment at the assembly line. Waiting time was $44 \mathrm{~s}$ and the pure working time was $82 \mathrm{~s}$. At the production line cycle time is $20 \mathrm{~s}$ and we could inspect only every seventh work-piece.

With the first parallel algorithm the waiting time dropped to $50 \%(17-19 \mathrm{~s})$ and the wavelet analysis time dropped by $40 \%$ (54-56 s). This algorithm presented a hopeful result because every second or third work-piece could be inspected. With an established three-work-piece hardware the measurement time is the same as the production line cycle time.

With the improved parallel algorithm some time was saved again. These developments improved the efficiency of the repeats and decreased the idle times. With direct hardware controlling functions only smaller inspection parts were repeated. The average working time reached 39-41 s, and the idle time shortened to 8-9 s.

Without repeats inspections are simple and every work-piece is inspected only once. In laboratory environment this minimal inspection time was reached (see Fig. 6).

The sequential method completes it in $40 \mathrm{~s}$. The parallel algorithm in this case decreased the idle time from $16 \mathrm{~s}$ to $6-7 \mathrm{~s}$ and with the improved algorithm to $4 \mathrm{~s}$. The minimal inspection time with the final improved algorithm is $22-24 \mathrm{~s}$.

In industrial environment depending on the noise level the minimal inspection time is between the minimal and the maximal threshold results. 
Average cycle time (max, with repeats)

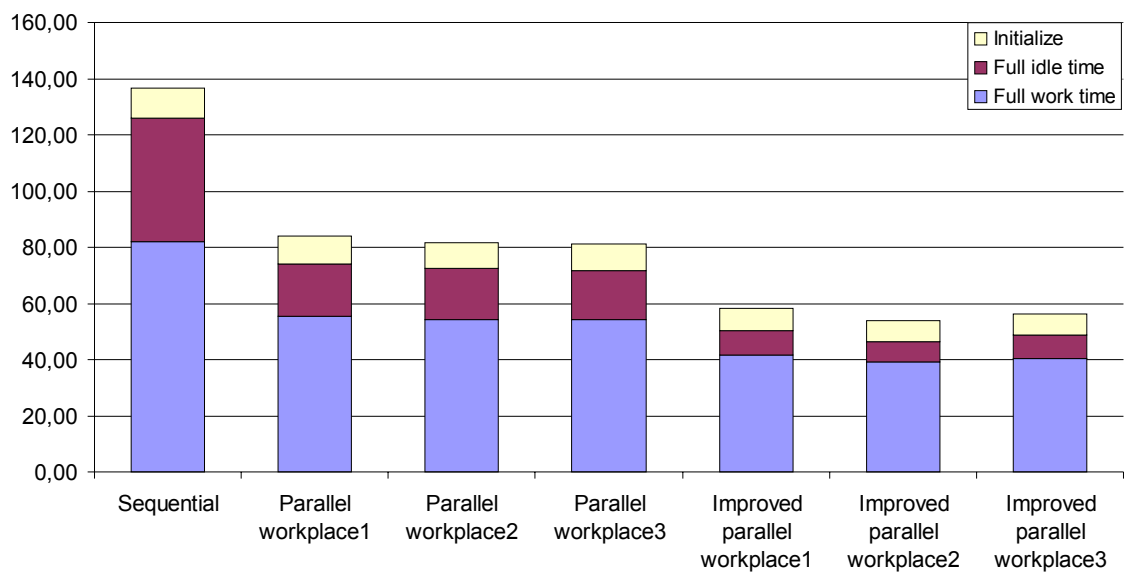

Fig. 5. Average running time of processing with three repeats

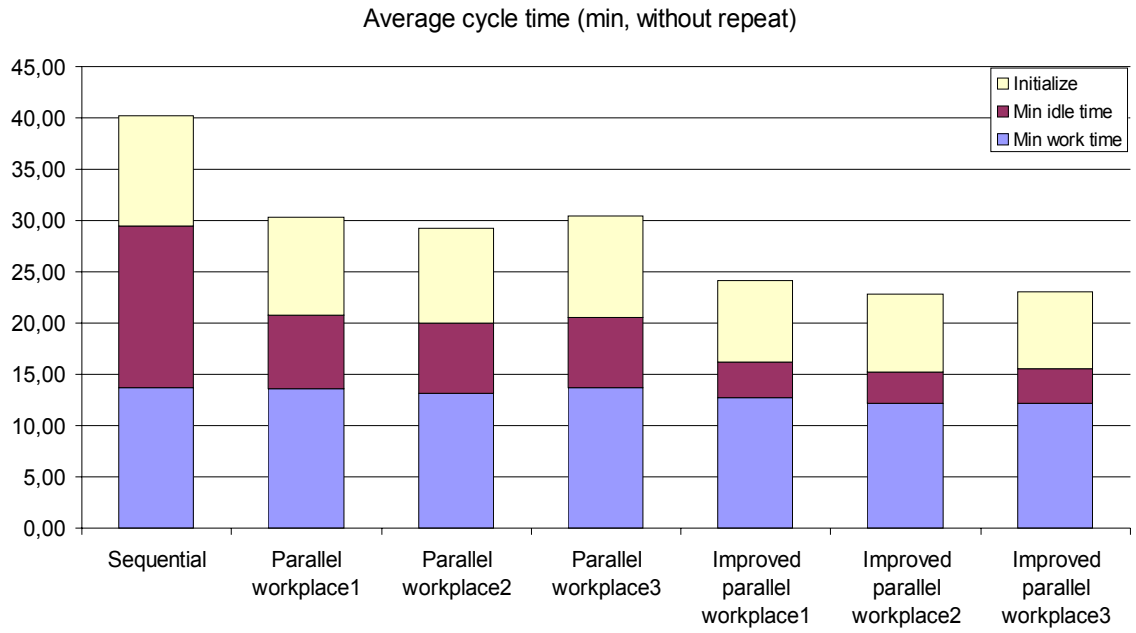

Fig. 6. Average simple inspection running time without repeats

\section{Summary}

The developed modular precision noise analyzer is a specialized tool to be used on a high noise level production line. The program has a client for the industrially

Pollack Periodica 3, 2008, 3 
specialized hardware. The communication is asynchronous and the client carries out interactive operations with the hardware. In case of false or noisy measurements when the quality class cannot be determined, the program can restart phases of the inspection. The program is usable on high-speed industrial production lines as a complex quality control station. The process of high precision noise inspection demands high processor and time capacity. For further research we have to optimize the analyzer algorithms.

\section{Acknowledgements}

This work was supported by OTKA No T043258 and by GVOP-3.2.2.-2004-07-0020/3.0 projects.

\section{References}

[1] Wang Y. S., Lee C. M., Kim D. G., Xu Y. Sound-quality prediction for non-stationary vehicle interior noise based on wavelet pre-processing neural network model, Journal of Sound and Vibration, Vol. 299, No. 4-5, February, 2007, pp. 933-947.

[2] Shtub A. On the natural cycle time of assembly lines, Journal of Operations Management, Vol. 5, No. 1, 1984, pp. 19-25.

[3] Boysen N., Fliedner M. A versatile algorithm for assembly line balancing, European Journal of Operational Research, Vol. 184, No. 1, 2008, pp. 39-56.

[4] Steinbuch M., Weiland S., Singh T. Design of noise and period-time robust high-order repetitive control, with application to optical storage, Automatica, Vol. 43, No. 12, 2007, pp. 2086-2095.

[5] Fastl H., Zwicker E. Psychoacoustics, facts and models, Springer, 1999, pp. 111-149.

[6] Halim E. B., Choudhury S., Sirish L. S., Zuo M. J. Time domain averaging across all scales, A novel method for detection of gearbox faults, Mechanical Systems and Signal Processing, Vol. 22, No. 2, 2008, pp. 261-278.

[7] Upton R. et al. Use of real-time Zwicker loudness in automotive noise measurements, JSAE Review, Vol. 15, No. 3, 1994, pp. 188-191.

[8] Pintér I. Noise suppression using non-linear speech model, Pollack Periodica, Vol. 2, Supplement 1, 2008, pp. 121-133. 